\title{
Experiences of the Emergency Department at the Pyeongchang Polyclinic During the 2018 PyeongChang Winter Olympic Games
}

\author{
Kwangmin Kim², Ji Young Jang, ${ }^{2,3}$ Gilseong Moon², Hongjin Shim ${ }^{2,3}$, Pil Young Jung ${ }^{2,3}$, \\ Sungyup $\mathrm{Kim}^{2,3}$, Young Un Choi ${ }^{2,3}$, and Keum Seok Bae ${ }^{2,3}$ \\ ${ }^{1}$ Department of Surgery, Saidabad Clinic, Dhaka, Bangladesh. \\ ${ }^{2}$ Department of Surgery, Yonsei University Wonju College of Medicine, Wonju; \\ ${ }^{3}$ Trauma Center, Wonju Severance Christian Hospital, Wonju, Korea.
}

Purpose: The 2018 PyeongChang Winter Olympic Games involved 2925 elite athletes, and providing proper health care services for these elite athletes was a critical priority. We established an emergency department (ED) in the Pyeongchang Mountain Polyclinic during the Olympics, which served staff and athletes from many countries. This experience, as well as a description of illnesses and injuries encountered during the games, may provide useful information for planning medical care at similar events in the future. Materials and Methods: The polyclinic ED operated from January 25 to February 27, 2018. All cases were enrolled in this study, and their data were analyzed by date and category. In addition, the number of injuries by body part, number of illnesses by organ system, and illness symptoms and causes were analyzed.

Results: In total, 288 patients were encountered in the ED. These included 113 injuries and 175 illnesses. We consulted with 153 staff members and 75 athletes, and reported that the fingers were the most commonly injured body part, followed by the knee. The respiratory system was the most commonly involved organ system, and the most common cause of illness was infection. Thirtyeight influenza tests were performed, among which the results of seven were positive. We performed 17 norovirus tests, among which the results of four were positive.

Conclusion: Our analysis of our ED experience will aid arrangements for medical services in future Winter Games. Additionally, given our new experience, we will now be able to provide better medical services for future winter sports events.

Key Words: 2018 PyeongChang Winter Olympic Games, polyclinic, emergency department

\section{INTRODUCTION}

The 2018 PyeongChang Winter Olympic Games hosted 2925 elite athletes from 93 National Olympic Committees. A critical part of the Olympic Games is to provide proper health care ser-

Received: December 14, 2018 Revised: February 27, 2019

Accepted: March 6, 2019

Corresponding author: Keum Seok Bae, MD, PhD, Department of Surgery, Yonsei University Wonju College of Medicine, 20 Ilsan-ro, Wonju 26426, Korea. Tel: 82-33-741-0573, Fax: 82-33-742-0574, E-mail: bksgs@yonsei.ac.kr

-The authors have no potential conflicts of interest to disclose.

(C) Copyright: Yonsei University College of Medicine 2019

This is an Open Access article distributed under the terms of the Creative Commons Attribution Non-Commercial License (https://creativecommons.org/licenses/ by-nc/4.0) which permits unrestricted non-commercial use, distribution, and reproduction in any medium, provided the original work is properly cited. vices. In fact, protection of the health of competing athletes is a key objective during the Olympic Games and forms an important part of the International Olympic Committee's (IOC's) agenda. ${ }^{1-3}$ One of the main aims of the IOC is to provide freely available healthcare to all athletes during the Olympic Games, as well as to institute safeguarding measures to protect athletes during competition. ${ }^{3}$ As part of this, in cases of injury or illness, comprehensive medical services must be provided to athletes and their respective national delegations. ${ }^{4}$ Comprehensive medical services consisted of several parts, including a medical office at each venue, the Olympic hospital, and the polyclinic at the Olympic Village. Trauma-dedicated attendings at the Wonju Severance Christian Hospital (WSCH) served as the emergency department (ED) in the Pyeongchang Mountain Polyclinic for the period covering the Olympics, serving staff and 
athletes from many countries. To ensure our services were sufficient, staff in charge of health care in each Olympic committee visited our hospital to check the treatment process, including registration and payment. Our experiences with running the polyclinic, as well as a description of illnesses and injuries encountered, could provide useful information for medical care planning at similar events in the future.

\section{MATERIALS AND METHODS}

\section{The 2018 Winter Olympic Games polyclinic}

Snow sport competitions took place in Pyeongchang, while ice sports occurred in Gangneung. The Taebaek Mountains are located between these two regions, which are located approximately $60 \mathrm{~km}$ apart. Therefore, two polyclinics were operated at each Olympic Village (Pyeongchang and Gangneung). The trauma-dedicated staff served the Pyeongchang polyclinic, which had two floors. The ED was located on the right side of the first floor and had a separate entrance from that of the outpatient clinic (Fig. 1).

The Pyeongchang polyclinic ED was staffed with salaried employees from their regular positions, mostly from WSCH. Two orthopedic surgeons were from Severance Hospital in Seoul. Work was divided into shifts to provide uninterrupted ED management coverage 24 hours a day. Two trauma surgeons worked in the ED for 24 hours alternatively every two days, three emergency physicians covered the ED for 24 hours every three days, and three orthopedic surgeons were assigned to each of three shifts for 24 hours. Seven nurses worked on a three-shift system. A total of four emergency medical technicians worked for 24 hours every four days as a roster. In addition, the assistant director of the polyclinic, who was a trauma surgeon, remained at the ED from 7 am to $11 \mathrm{pm}$ to manage patients or administrative services.

The Olympic hospitals, WSCH and Gangneung Asan Hospital (GAH), were located near the polyclinic. Gangneung Medical Center (GMC) was also available, particularly for patients needing magnetic resonance imaging (MRI) services. Severely injured patients were transferred to WSCH, as it had a regional trauma center. Ground and helicopter transportation were available: two ambulances were always available at the polyclinic, and four helicopters were available for emergency patients. One helicopter was posted around the Jeongseon Alpine Centre, and two remained at the Jinbu helicopter station near Pyeongchang mountain cluster (Fig. 2). The fourth was a doctor helicopter belonging to WSCH that stayed at the WSCH trauma center.

An electronic medical records system was used for all patients. When X-rays and medicines were ordered, the order slip printed out directly in the X-ray room or pharmacy, respectively, instead of being delivered by hand. The laboratory was open from 7 am to $11 \mathrm{pm}$, which was separate from the doping

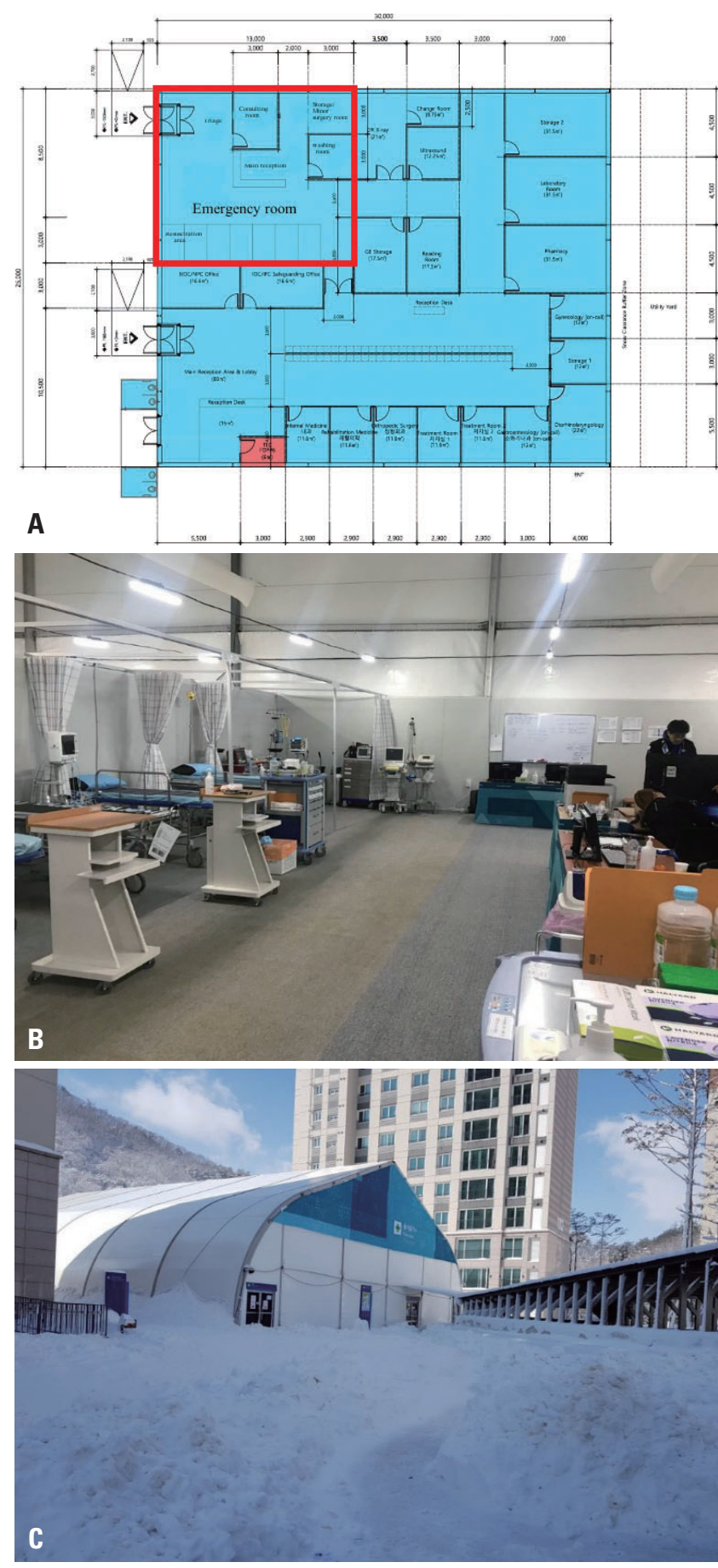

Fig. 1. ED of Pyeongchang polyclinic. (A) Cross-sectional diagram of the polyclinic (red box indicates ED at the polyclinic), (B) inside the polyclinic $E D,(C)$ exterior of the polyclinic. ED, emergency department.

test laboratory. Complete blood count, chemistry, urine analysis, cardiac enzyme, influenza A and B antigen, and norovirus rapid tests were available. The pharmacy was also open during this time, and basic and emergency medicine was available in the ER 24 hours a day.

The main drawback of the polyclinic was the lack of computed tomography (CT) and MRI services. Therefore, patients 


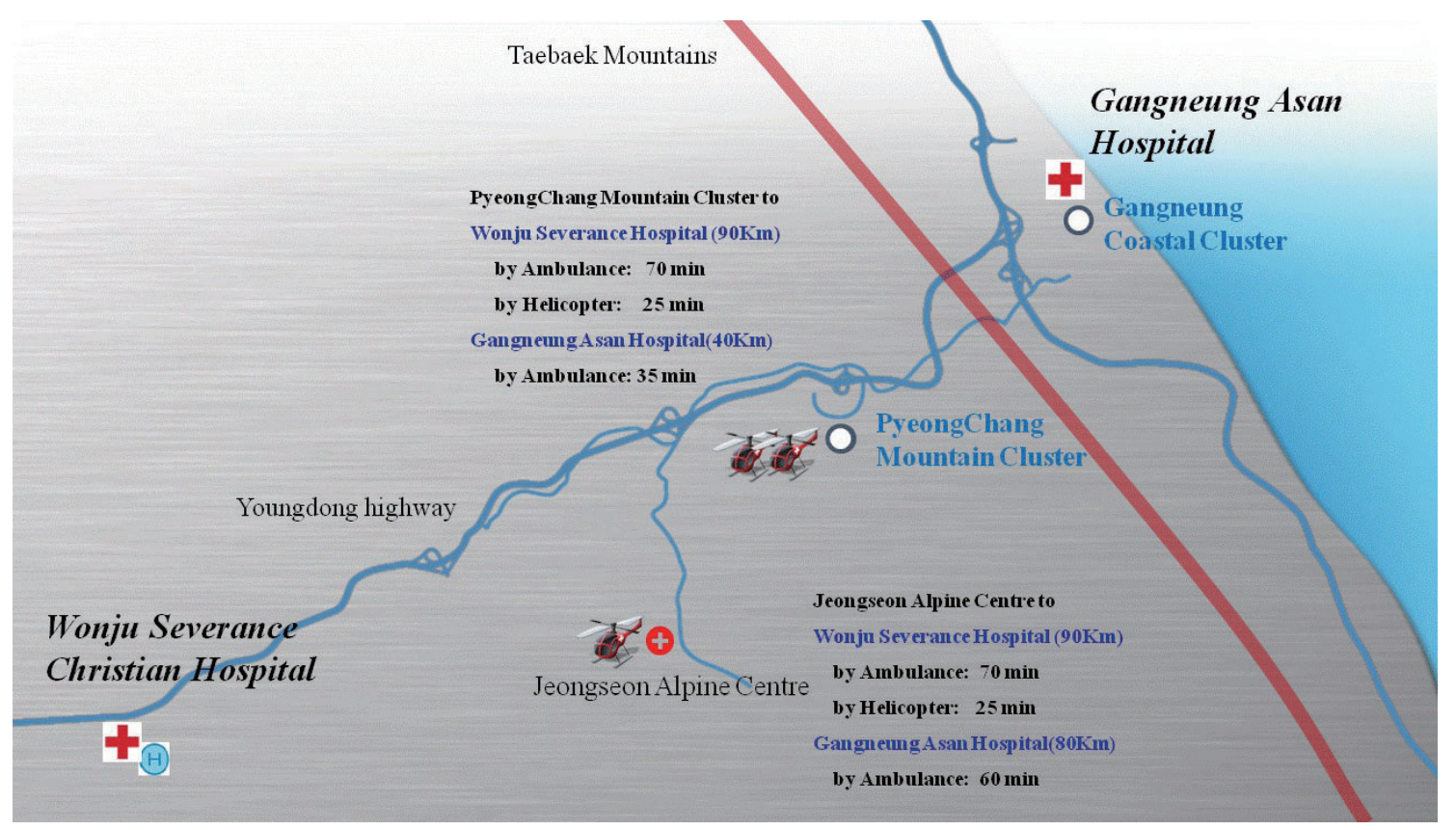

Fig. 2. Geographic location of Olympic hospitals and helicopter stations.

who needed a CT scan were transferred to GAH, and patients needing MRI were transferred to GMC. Booking for patients who needed CT or MRI services was arranged at the ED. Available radiologists performed X-ray and ultrasonography (US) services. US services were performed in the ED; therefore, emergency US, including extended focused assessment with sonography for trauma, was available for every incident.

\section{Data collection}

We retrieved all medical records on all patients treated for injuries and illnesses in the Pyeongchang polyclinic from the $\mathrm{Py}$ eongchang Organizing Committee for the 2018 Winter Olympic Games' medical staff. Concurrently, the same information during the Olympic Games was collected from our own data recorded by polyclinic nurses. The ED operated from January 25 to February 27. Therefore, all cases during the period were enrolled in this study.

For every patient visiting the ED, an electronic chart was created, and demographic characteristics (accreditation number, age, sex, birth date, home country), sports/events, main symptoms, history, illness or injury diagnosis, treatment, and type of discharge were recorded. In cases of injury, location, type, and cause were recorded. In cases of illness, affected systems, symptoms, causes, and estimated time loss were recorded. Every evening, a medical record administrator verified that all the charts were correctly completed and sent them to the chief medical officer. ED nurses also recorded basic demographics, main symptoms, treatment, and type of discharge for our own convenience.

\section{Definitions}

An injury was defined as new (pre-existing, not fully rehabili- tated conditions were not recorded) or recurring musculoskeletal symptoms, concussions, or internal organ injury. An illness was defined as any physical complaint (not related to injury) newly developed that received medical attention. If multiple body parts were injured during the same incident, multiple types of injuries occurred in the same body part, or if different body parts were affected by illnesses, every injury/illness was investigated for this study. The category was classified as staff, athlete, other (e.g., spectator, visitor, service company worker), and unknown (not recorded). Environmental cause was defined as the occurrence of symptoms due to the inability of the body to adapt to rapid environmental changes without evidence of infection.

\section{Ethical approval}

All information was treated with strict confidence, and our medical database was anonymized for use in this study. This study was approved by Wonju Severance Christian Hospital Institutional Review Board (IRB No: CR318040).

\section{Data analysis}

Cases were analyzed by date and category. In addition, we analyzed the number of injury encounters by body part, number of illness encounters by organ system, and symptoms and causes. Descriptive statistics were used for this study. All data were statistically analyzed using SPSS (SPSS for Windows, versions 18.0 SPSS Inc., Chicago, IL, USA).

\section{RESULTS}

A total of 288 patients were encountered in the ED: 113 had 
injuries and 175 had illnesses. The patients comprised 180 men and 108 women. The number of cases tended to increase until January 31. From February 1 to the opening of the Olympic Games, the daily numbers of patients encountered were similar. However, once the Olympic Games began, the number of patients tended to increase. In particular, 22 patients were reported on February 25, the highest number of patients by date (Fig. 3).

According to the analysis of all patients by category, 153 patients (53.1\%) were staff members and 75 (26.0\%) were athletes. Of all 113 injury cases, 61 patients (54.0\%) were staff members and $34(30.1 \%)$ were athletes (Fig. 4$)$. When we analyzed the injury cases by body part, we found that the most common injuries among staff members were in the fingers (14 cases) and hands (13 cases). Among athletes, the knee was the most commonly injured body part ( 8 cases), followed by the ankle ( 4 cas- es) (Table 1). Multiple trauma was present in eight cases (7.1\%). Hand and finger lacerations were the most common in four cases. There were three cases of head and chest contusions, and one case of elbow and knee injury due to non-contact trauma.

The respiratory system was the most commonly involved system for all categories: staff members accounted for 45 patients and athletes accounted for 26 patients. The gastrointestinal tract was the second most commonly affected system for all categories: 28 cases occurred in staff and seven cases occurred in athletes (Table 1). The most common symptom that patients complained of was pain, with 49 cases reported in staff members, 24 in athletes, and 14 and 11 in the other and unknown categories, respectively. The second most common symptoms were respiratory symptoms (72 cases), including dyspnea, cough, and sputum. Symptoms associated with the gastrointestinal tract, including diarrhea, vomiting, and dys-

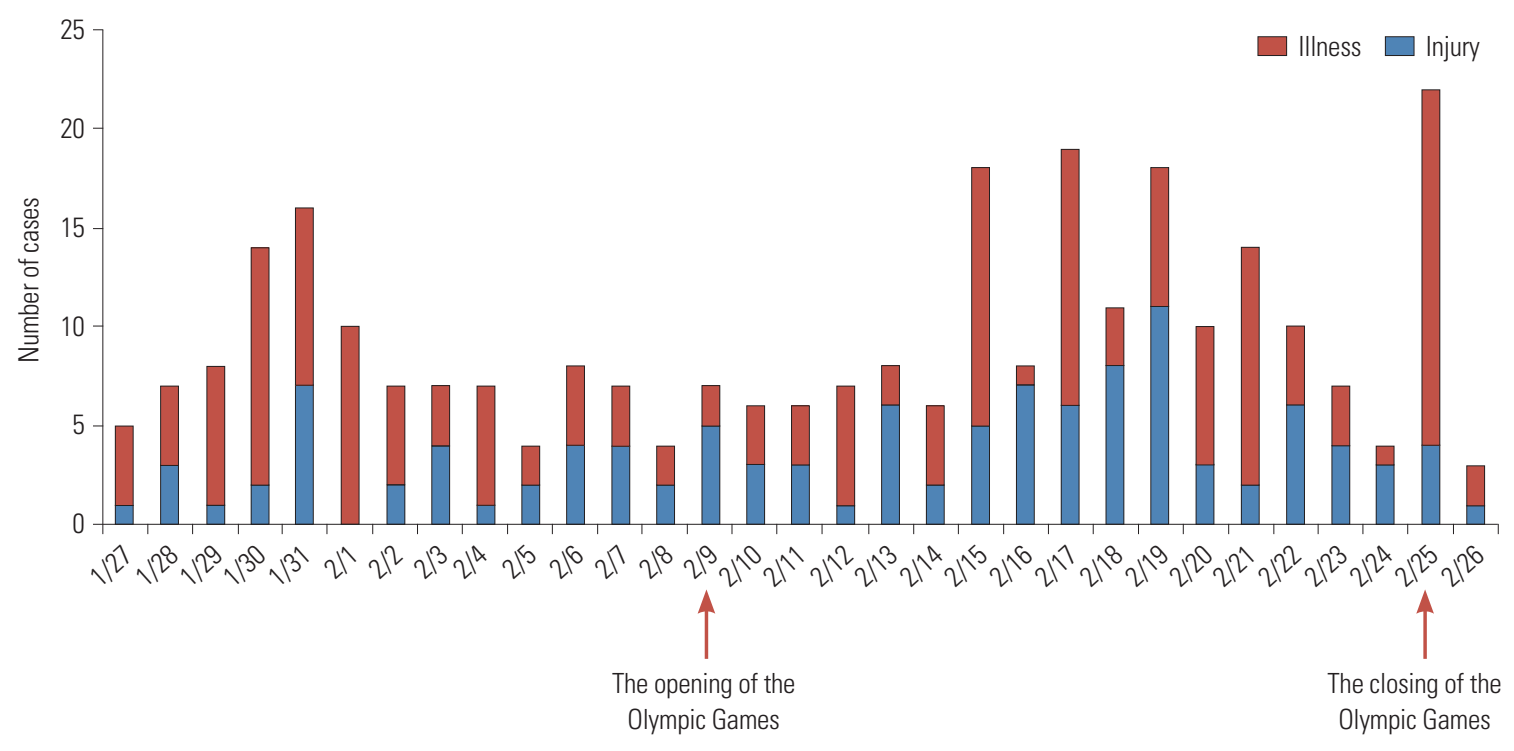

Fig. 3. Emergency department encounters by date.
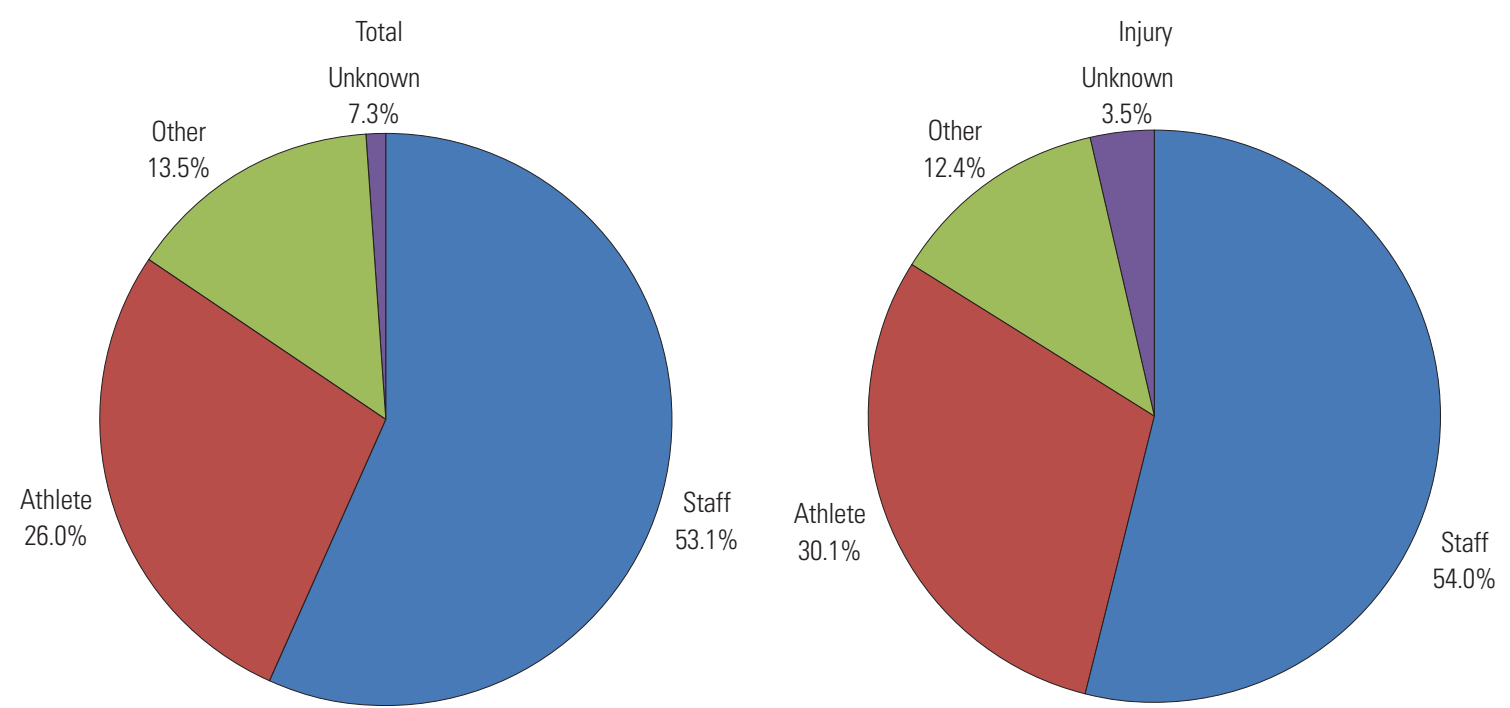

Fig. 4. Emergency department encounters by category. 
pepsia, were the next most common symptoms (37 cases) (Table 1). For all categories, the most common cause of illness was infection (119 cases), followed by illness caused by environmental effects (35 cases) (Table 1 ).

The total number of transports to hospitals was 67, split between GMC (for MRI) (47 cases), GAH (16 cases), and WSCH (4 cases). Influenza tests were issued in 38 cases, among which seven patients tested positive. A norovirus rapid test and polymerase chain reaction (PCR) was performed in 17 cases, among which four patients (all athletes) tested positive.

\section{DISCUSSION}

The main drawback of the Pyeongchang polyclinic was the lack of CT or MRI services. Three strategies were used to overcome this problem. First, the assistant director remained at the ED to arrange booking and transportation to hospitals from 7 am to $11 \mathrm{pm}$. Second, the MRI booking and insurance checking processes were simplified by sharing a regular form containing patient details with the social network service (SNS), thereby eliminating the need for telephone communication. In cases wherein patients were transported to the Olympic hospitals (GAH or WSCH), the SNS was quite useful. BAND (Camp mobile, Seoul, South Korea) was used to communicate with each venue, GMC, and GAH (Supplementary Fig. 1, only online), while Kakaotalk (Kakao Corporation, Jeju, South Korea) was used to communicate with WSCH. Third, the drive from the polyclinic to GMC generally took 30 minutes, and two ambulances remained near the polyclinic to transport patients quickly.

The fingers and hands were the most commonly injured body parts among staff members, as they performed physical work requiring them to use their hands. For athletes, the most commonly injured body parts were the knee and ankle. Events around the Pyeongchang polyclinic were snow sports, including ski jumping, mogul skiing, alpine skiing, and snowboarding, all of which require the knees and ankles to absorb shock. Similar to our experience, the knees were the most common injury location among alpine skiing and snowboarding athletes during the 2010 Vancouver Winter Olympic Games. ${ }^{5}$

In patients who visited the ED with illnesses, the respiratory tract was the most commonly involved system, followed by the gastrointestinal tract. Cold weather and a sudden change in surroundings might influence these organ systems, resulting in a greater number of cases involving these systems. In addition, infection was the most common cause of illness. This high incidence of respiratory infections mirrors data from other elite sporting events, ${ }^{6,7}$ is often related to infections, and is typical of winter events and not summer events. ${ }^{8-10}$

The number of daily patients tended to increase toward the end of the Olympic Games, likely because more competitions were held during this time. This trend is similar to that reported
Table 1. Number of Injured Body Parts and IIIness-Affected Systems, Symptoms, and Causes by Category

\begin{tabular}{lrrrrr}
\hline & Staff & Athlete & Other & Unknown & All \\
\hline $\begin{array}{l}\text { Injuries } \\
\text { Injury location }\end{array}$ & & & & & \\
\hline Face & 2 & 2 & 0 & 0 & 4 \\
\hline Head & 3 & 1 & 0 & 0 & 4 \\
\hline Thoracic spine/upper back & 9 & 1 & 0 & 0 & 10 \\
\hline Sternum/ribs & 2 & 2 & 0 & 0 & 4 \\
\hline Lumbar spine/lower back & 2 & 3 & 1 & 0 & 6 \\
\hline Abdomen & 0 & 1 & 0 & 0 & 1 \\
\hline Pelvis/sacrum/buttocks & 0 & 1 & 0 & 0 & 1 \\
\hline Shoulder/clavicle & 4 & 1 & 0 & 0 & 5 \\
\hline Elbow & 0 & 1 & 0 & 1 & 2 \\
\hline Forearm & 0 & 2 & 0 & 0 & 2 \\
\hline Wrist & 1 & 1 & 0 & 0 & 2 \\
\hline Hand & 13 & 0 & 6 & 0 & 19 \\
\hline Finger & 14 & 1 & 2 & 2 & 19 \\
\hline Hip & 0 & 1 & 0 & 0 & 1 \\
\hline Groin & 0 & 1 & 0 & 0 & 1 \\
\hline Thigh & 0 & 1 & 0 & 0 & 1 \\
\hline Knee & 3 & 8 & 1 & 1 & 13 \\
\hline Lower leg & 7 & 2 & 1 & 0 & 10 \\
\hline Achilles tendon & 1 & 0 & 0 & 0 & 1 \\
\hline Ankle & 1 & 4 & 0 & 0 & 5 \\
\hline Foot/toe & 5 & 0 & 1 & 0 & 6 \\
\hline Other & 1 & 0 & 1 & 0 & 2 \\
\hline Ses & & & & & \\
\hline
\end{tabular}

Illnesses

IIIness-affected system

$\begin{array}{llllll}\text { Gastrointestinal } & 28 & 7 & 7 & 4 & 46\end{array}$

$\begin{array}{llllll}\text { Respiratory } & 45 & 26 & 16 & 10 & 97\end{array}$

Allergic

Dermatologic

Urogenital

Gynecological

Cardiovascular

Neurological

Musculoskeletal

Dental

Eye

Other

200002

$\begin{array}{lllll}1 & 0 & 0 & 0 & 1\end{array}$

Illness symptom

$\begin{array}{llllll}\text { Fever } & 8 & 6 & 2 & 1 & 17\end{array}$

$\begin{array}{llllll}\text { Pain } & 49 & 24 & 14 & 11 & 98\end{array}$

$\begin{array}{llllll}\text { Diarrhea, vomiting, dyspepsia } & 22 & 7 & 5 & 3 & 37\end{array}$

$\begin{array}{llllll}\text { Dyspnea, cough, sputum } & 35 & 17 & 13 & 7 & 72\end{array}$

Other (including

$\begin{array}{llllll}\text { dehydration, anaphylaxis, } & 8 & 2 & 1 & 0 & 11\end{array}$

lethargy, dizziness)

IIIness cause

$\begin{array}{lrrrrr}\text { Infection } & 56 & 32 & 17 & 14 & 119 \\ \text { Environmental } & 23 & 0 & 8 & 4 & 35 \\ \text { Exercise-induced } & 1 & 3 & 0 & 0 & 4 \\ \begin{array}{l}\text { Other (including } \\ \text { pre-existing, drug) }\end{array} & 13 & 6 & 2 & 1 & 22\end{array}$


at the polyclinic during the 2002 Salt Lake City Winter Olympic Games. ${ }^{4}$ Additionally, respiratory diseases also increased in frequency among staff and athletes, who were living in cold and unfamiliar surroundings. In particular, the largest number of patients was recorded on February 25. During this time, one of the USA athletes was confirmed to have influenza, and following that visit, 15 of the closest contacted individuals visited to be tested and receive treatment.

Specific viral infections encountered at the polyclinic were influenza and norovirus infection. Influenza rapid test kits were available in the ED every day, although the laboratory was closed from $11 \mathrm{pm}$ to $7 \mathrm{am}$. Tamiflu (Roche, Basel, Switzerland) and Peramiflu (Green Cross, Yongin, South Korea) injections for patients who experienced gastrointestinal troubles with Tamiflu were also prepared at the ED. Norovirus was confirmed in 168 staff members who stayed at an accommodation near Pyeongchang for work forces during the Olympics. We prepared some strategies to prevent the spread of the norovirus to the Olympic Village. First, the head of infection control and two nurses at WSCH rushed to the polyclinic and took charge of infection control in the polyclinic. One nurse from Pyeongchang Public Health Center and three staff members from the Korea Centers for Disease Control and Prevention (KCDCP) also visited the polyclinic. Second, suspected patients were carefully controlled through the treatment process, and the guidelines for treating patients with suspected norovirus were distributed. Suspected patients were isolated at their accommodations rather than instructing them to visit the polyclinic. So-called norovirus busters, consisting of an emergency physician or the trauma surgeon on duty, a nurse from the public health center, and one staff member from the KCDCP visited their accommodations to check patient conditions, obtain specimens, and distribute medicines if desired or needed. A total of two specimens per patient were obtained by anal swabbing. One was sent to the laboratory in the polyclinic to undergo rapid testing, and the second was sent to the KCDCP for a norovirus PCR because of the low sensitivity and specificity of the rapid test. The PCR result was reported within about six hours after the PCR was initiated. Despite these preparations, a Swiss player who participated in the skiing game was confirmed to be the first norovirus-infected patient in the athlete's village. We did our best to prevent spread through the above strategy and thorough isolation. As a result, only four patients were confirmed to have norovirus at the Olympic Village, limiting its spread.

Remembering an accident during the Vancouver Winter Olympics, during which one elite athlete passed away in a luge practice run, some strategies were prepared for severely injured patients. First, a trauma-dedicated surgeon in WSCH remained at the ED every day, and devices (e.g., electrocautery, suction device) for performing surgery, including damage control surgery (intraperitoneal tape packing and preperitoneal pelvic packing) were prepared and stored ready for use. In addition, portable ventilators, monitoring devices, defibril- lators, and rapid infusion pumps, among others, were prepared, which were essential for managing severely injured patients before transportation. Therefore, proper treatment by a trauma surgeon was possible at the ED. Second, helicopters (three emergency helicopters and one doctor helicopter) for fast transportation were prepared for severely injured patients, eliminating the need for ground transportation. Gangwon Province is a mountainous region, and the regional trauma center at WSCH is $90 \mathrm{~km}$ away from the Pyeongchang polyclinic. In addition, traffic was a significant concern during the Olympics since only one four-lane highway (Youngdong Highway) is available for ground transportation from Pyeongchang to Wonju. Fortunately, there was no need for helicopter transportation during that period as no unstable patients visited the polyclinic. Orthopedic surgeons also remained at the ED every day because musculoskeletal trauma was expected to be the major cause of all traumas. However, we covered all trauma patients, from trivial patients to severely injured patients.

One of the drawbacks of our management of the polyclinic ED was the lack of a simulation for the staff before the ED initiated operations, resulting in the medical staff being unfamiliar with the work. For medical services in the 2010 Vancouver Olympic Winter Games, the training program for medical staff was a four-phase education and training curriculum, designed to foster the performance of a high-functioning interdisciplinary medical team. Fifty-five percent of physicians $(n=10)$ who participated in this simulation training provided positive feedback and felt they received adequate simulation training. Thirty-nine percent of physicians $(\mathrm{n}=7)$ recommended that similar training schedules should be implemented to improve familiarity with working at the mobile medical unit/polyclinic. ${ }^{11}$ In our case, several meetings with the national Olympic Organizing Committee were held to improve the quality of medical services. In addition, test events were held, during which comprehensive medical services were provided. However, it would have been preferable to have a polyclinic operating simulation before opening the Olympic Games to provide familiarity with the working conditions and reduce errors.

The retrospective design could be a limitation of this study. However, these data are valuable because they were collected in cooperation with the IOC research center. At these Olympic Games, the polyclinics were divided into two areas (Pyeongchang and Gangneung), and the patient data of the ED in Gangneung polyclinic could not be used because they were operated in other hospital. However, there is a high possibility of serious trauma in snow and sled events, and the experience of operating the Pyeongchang polyclinic ED, which treated the athletes participating in these events, is meaningful.

In summary, the analysis and experience of the cases encountered at the ED, including resolution of unexpected problems, such as norovirus infection, will be helpful for planning medical services for future Olympic Winter Games. Pyeongchang will likely become a mecca for Asian winter sports. In addition, 
Pyeongchang may become an important place, bringing South and North Korea together once more by hosting the upcoming 2021 Winter Asian Games. During this time, our hospital will be a provider of medical services, and we will be able to provide better medical services based on this experience for all future winter sporting events in Gangwon Province.

\section{ACKNOWLEDGEMENTS}

We wish to express our gratefulness to the Head of the NOC Medical Department, Hanseong Lee, and the Chief Manager of the Pyeongchang Polyclinic, Yeonnam Yoo, for their strong support. We thank Younghee Lee, Director of Wonju Severance Christian Hospital, Yonsei University College of Medicine and CMO of the Olympic games, for his very important support. Also, we would like to extend our thanks to Sejoon Kim, National Team doctor and orthopedic surgeon, for inspiring us to write this study. We also thank all the workers in the ED at the polyclinic.

\section{AUTHOR CONTRIBUTIONS}

Conceptualization: Kwangmin Kim, Ji Young Jang, Hongjin Shim. Data curation: Kwangmin Kim. Formal analysis: Kwangmin Kim. Investigation: Kwangmin Kim. Methodology: Kwangmin Kim, Ji Young Jang. Project administration: Kwangmin Kim, Ji Young Jang, Gilseong Moon, Hongjin Shim, Pil Young Jung, Sungyup Kim, Young Un Choi, Keum Seok Bae. Resources: Kwangmin Kim, Ji Young Jang, Gilseong Moon, Hongjin Shim, Pil Young Jung, Sungyup Kim, Young Un Choi, Keum Seok Bae. Software: Kwangmin Kim, Ji Young Jang. Supervision: Keum Seok Bae. Validation: Kwangmin Kim, Ji Young Jang, Gilseong Moon, Hongjin Shim, Pil Young Jung, Sungyup Kim, Young Un Choi, Keum Seok Bae. Visualization: Kwangmin Kim, Ji Young Jang. Writing_original draft: Kwangmin Kim. Writing_review \& editing: Kwangmin Kim, Ji Young Jang, Gilseong Moon, Hongjin Shim, Pil Young Jung, Sungyup Kim, Young Un Choi, Keum Seok Bae.

\section{ORCID iDs}

Kwangmin Kim Ji Young Jang Gilseong Moon https://orcid.org/0000-0003-0496-1303 https://orcid.org/0000-0001-6372-4194 https://orcid.org/0000-0002-5573-8585
Hongjin Shim Pil Young Jung Sungyup Kim Young Un Choi

Keum Seok Bae

https://orcid.org/0000-0003-3073-3806 https://orcid.org/0000-0001-6460-8072 https://orcid.org/0000-0003-3006-8391 https://orcid.org/0000-0003-2410-7788 https://orcid.org/0000-0003-4728-3047

\section{REFERENCES}

1. Ljungqvist A, Jenoure P, Engebretsen L, Alonso JM, Bahr R, Clough A, et al. The International Olympic Committee (IOC) Consensus Statement on periodic health evaluation of elite athletes March 2009. Br J Sports Med 2009;43:631-43.

2. Steffen K, Soligard T, Engebretsen L. Health protection of the Olympic athlete. Br J Sports Med 2012;46:466-70.

3. Vanhegan IS, Palmer-Green D, Soligard T, Steffen K, O'Connor P, Bethapudi S, et al. The London 2012 Summer Olympic Games: an analysis of usage of the Olympic Village 'Polyclinic' by competing athletes. Br J Sports Med 2013;47:415-9.

4. Reeser JC, Willick S, Elstad M. Medical services provided at the Olympic Village polyclinic during the 2002 Salt Lake City Winter Games. WMJ 2003;102:20-5.

5. Engebretsen L, Steffen K, Alonso JM, Aubry M, Dvorak J, Junge A, et al. Sports injuries and illnesses during the Winter Olympic Games 2010. Br J Sports Med 2010;44:772-80.

6. Ruedl G, Schobersberger W, Pocecco E, Blank C, Engebretsen L, Soligard T, et al. Sport injuries and illnesses during the first Winter Youth Olympic Games 2012 in Innsbruck, Austria. Br J Sports Med 2012;46:1030-7.

7. Soligard T, Steffen K, Palmer-Green D, Aubry M, Grant ME, Meeuwisse W, et al. Sports injuries and illnesses in the Sochi 2014 Olympic Winter Games. Br J Sports Med 2015;49:441-7.

8. Weiss BP, Mascola L, Fannin SL. Public health at the 1984 Summer Olympics: the Los Angeles County experience. Am J Public Health 1988;78:686-8.

9. Thompson JM, Savoia G, Powell G, Challis EB, Law P. Level of medical care required for mass gatherings: the XV Winter Olympic Games in Calgary, Canada. Ann Emerg Med 1991;20:385-90.

10. Piat SC, Minniti D, Traversi D, Gianino MM, Massazza G, Siliquini R. Torino 2006 Winter Olympic Games: highlight on health services organization. J Emerg Med 2010;39:454-61.

11. Brown DR, Heidary B, Bell N, Appleton L, Simons RK, Evans DC, et al. Creating a gold medal Olympic and Paralympics health care team: a satisfaction survey of the mobile medical unit/polyclinic team training for the Vancouver 2010 winter games. BMC Res Notes 2013;6:462. 